\title{
ANALYSIS OF NON-STOP TRAFFIC AT ISOLATED SIGNALISED INTERSECTION BY MEANS OF DISCRETE EVENT SIMULATION
}

\author{
Al-Aomar, R. ; Alyousef, B. ${ }^{*} \&$ Al-Omari, B. H. ${ }^{* *}$ \\ * Industrial Engineering Department \\ ${ }^{* *}$ Civil Engineering Department \\ Jordan University of Science and Technology, Irbid-Jordan \\ E-Mail: ralaomar@just.edu.jo
}

\begin{abstract}
This paper includes a simulation-based analysis of non-stop traffic at isolated signalised intersections. A Min-Max traffic control method is proposed to replace conventional traffic signals and maintain a continuous traffic flow. The method applicability is tested by means of Discrete Event Simulation (DES) using AutoMod software package. A set of quantitative Measures of Performance (MOP) is used to demonstrate the benefits of the proposed method compared to conventional traffic signals. In addition to eliminating vehicles stoppages, simulation results of the proposed method showed improvement in the overall traffic flow by reducing vehicle average travel time, average delay time, and the average number of vehicles at the intersection. The proposed method can be adapted to suit other types of traffic intersections and flow patterns and conditions.

(Received in May 2007, accepted in October 2007. This paper was with the authors 2 months for 3 revisions.)
\end{abstract}

Key Words: Traffic Control, Discrete Event Simulation, Continuous Flow Intersections

\section{INTRODUCTION}

Traffic congestion is on the rise due to the continuing growth of urban areas, the increasing number of vehicles, and the increasing cost of building new roads. Therefore, it is necessary for researcher to explore creative and viable solutions to traffic problems especially at traffic intersections. Traffic signals are the most common traffic control system at both isolated and non-isolated intersections. The primary function of traffic signals is to assign right-of-way to conflicting movements of traffic at an intersection by means of time separation. This typically increases the traffic handling capacity of an intersection, improves the safety of both pedestrian and vehicular traffic, and reduces certain types of accidents.

Developing traffic control systems is based on a set of traffic-flow theories that investigate, model, and analyze the interactions among vehicles, drivers, and the transportation system [1]. A wide variety of traffic-related topics are discussed in [2] and [3]. Recent traffic studies are particularly focused on the effectiveness and continuity of vehicles flow at traffic intersections (also referred to as continuous flow intersection (CFI)) [4, 5, 6]. Traffic studies also assert that traffic-flow theory can be better understood and more easily characterized through advanced computation technology and simulation modelling [7, 8, 9]. Traffic simulation is a more efficient tool than traffic analysis in cases where mathematical analysis is infeasible due to flow complexity, cases when flow conditions change over time, cases when flow variability is significant, and cases when animation displays are needed to gain an understanding of system before its real implementation. Hence, traffic simulation software tools such as CORSIM, TRANSYT, and HCS are increasingly used to simulate and test traffic control schemes $[8,10,11]$. 
This study proposes a Min-Max traffic control system for preventing vehicles from stopping at isolated intersections. The study, however, utilizes Discrete Event Simulation (DES) to model traffic flow and validate the proposed traffic control systems. DES is a key tool in Industrial Engineering for system-level modelling in numerous production and business applications including manufacturing, banking, computer networks, call centres, and traffic simulation. The wide-range of DES applications can be mainly attributed to the tremendous improvement in simulation software tools in terms of ease-of-use, animation, and flexibility. Examples of common simulation packages include AutoMod, Arena, Witness, and Simul8. Details of DES methods, applications, and software tools can be found in [12], [13], and [14]. Still, however, using DES in traffic systems is limited to few applications as discussed in [15], [16], and [17].

In this paper, DES is utilized to validate the functionality of the proposed Min-Max method and to check its applicability in the real-world. DES is also used to compare the method performance to the commonly used two-phase traffic signal system by estimating a predefined set of Measures of Performance (MOP).

\section{SIGNALISED TRAFFIC INTERSECTIONS}

As discussed in [2], traffic signals assign the right-of-way to various traffic movements for different time intervals depending on traffic flow levels. Right-of-way is assigned by turning on a green signal for a certain length of time or an interval right-of-way is ended by a yellow change interval followed by the display of a red signal. The cycle length is the total time to complete one sequence of signalization around an intersection in an actuated controller unit. A complete cycle is dependent on the presence of calls on all phases. The intersection clearance interval provides vehicles with enough time at the end of yellow to clear before the next green is displayed.

Several methods are commonly used to control traffic signals at traffic intersections. A pre-timed signal control (fixed time signal) in which the signal assigns right-of-way at an intersection according to a predetermined schedule of the sequence of right-of-way (phases). The length of the time interval for each signal indication in the cycle is fixed based on historic traffic patterns. Such system is characterized by simplicity and the ability to be coordinated to provide continuous flow of traffic at a given speed along a particular route. A second type is traffic-actuated signal control of isolated intersections in which the green time is adjusted continuously in accordance with real-time measures of traffic to reduce delay. Demand is obtained from vehicle detectors placed on one or more of the approaches to the intersection.

Coordinated signals is another commonly used system for traffic control where a series of signalized intersections are designed to interact as a system so that groups of vehicles will be able to move through the signals without stopping. MUTCD (Manual on Uniform Traffic Control Devices) recommends that signals within $(0.81 \mathrm{~km})$ of each other be coordinated on major streets. Coordinated signalling, which runs on a common cycle time, is typically used along heavily travelled arterial streets with a frequent presence of traffic signals. The main disadvantage of signal coordination is that side street traffic has a longer wait time and the system may not reduce stops along a particular street due to capacity issues and complexity of the street system.

Finally, new smart traffic control system have emerged recently such as the Intelligent Vehicle Highway System (IVHS) in which vehicles and highways will exchange information about speed, acceleration, braking, obstructions, and road conditions through a two-way communication system. The automated highways will have a set of lanes on which vehicles with specialized sensors and wireless communications systems could travel under computer control at closely spaced intervals. Global Positioning System (GPS) offers an efficient and 
economic method to obtain precise coordinates and other related information, using the GPS satellite system.

An isolated intersection signal is one at which the timing is independent of any other signal in the region. Based on the [18], when timing isolated signals, short cycle lengths of 35-50 seconds are sufficient and longer lengths may be required at high-volume locations. The optimal cycle length permits traffic to pass the intersection with a minimum delay. The number of phases should also be kept to a minimum in order to minimize the total delay; the usual procedure is to implement two-phase operation. In general and based on [3], the minimum cycle length $C_{\min }$ in seconds for an $n$-phase signal is defined as follow:

$$
C_{\min }=\frac{\sum_{i=1}^{n} Y_{i}}{1-\left[\sum_{i=1}^{n} N_{i} S_{i} / 3600(P H F)\right]}
$$

Where:

$N \quad$ - Critical lane volume, expressed as the number of vehicles per lane in passenger car equivalents (pce) units entering the intersection during peak hour.

$Y \quad$ - Vehicle clearance interval (yellow time) in seconds for a road direction.

$S_{i} \quad$ - Approximated average headway between vehicles in a road direction.

PHF - Peak Hour Factor (the ratio of volume observed during peak hour to four times the maximum observed flow rate during 15-minute period).

It was found that the turning vehicles and commercial vehicles (buses and trucks) usually consume additional time to pass the intersection. These added delay times must be included in determining vehicle green intervals. Each left turn vehicle, right turn vehicle, and bus or truck was found to consume 1.56, 1.40, and 1.50 seconds of additional time, respectively. Thus, based on [3], the critical lane volume $N_{i}$ in terms of pce is computed as follows:

$$
N_{i}=\frac{T_{V i}+0.5 C_{i}+0.6 T_{L i}+0.4 T_{R i}}{n_{i}}
$$

Where:

$T_{V} \quad$ - Actual total traffic volume in a road direction.

$C \quad$ - Number of commercial vehicles for a road direction.

$T_{L} \quad$ - Number of left turns for a road direction.

$T_{R} \quad$ - Number of right turns for a road direction.

$n \quad$ - Number of usable lanes for a road direction.

In conventional traffic signals, the lower the time consumed by vehicles in yellow interval at intersection zone, the better the chance for arriving at a green light and passing without stopping. Hence, based on [19, 20], the yellow interval formula depends on velocity and distance and calculated as follows:

$$
Y_{i}=t+\frac{1}{2} \frac{v_{i}}{a}+\frac{\left(W_{i}+L_{i}\right)}{v_{i}}
$$

Where:

$t \quad$ - Perception reaction time of drivers.

$v \quad$ - Road speed in $\mathrm{m} / \mathrm{sec}$

a - Deceleration / acceleration rate of clearing vehicle.

W - Width of street crossed.

$L \quad$ - Length of vehicle. 


\section{PROPOSED SYSTEM (Min-Max method)}

Stopping at the sign persists to be the main problem of conventional traffic signals, which may stop and accumulate heavy flows to permit the crossing of minor movements in addition to increased pollution and noise. Along with that come the drivers' frustration due to multistopping, the risks of braking at high speed and running red lights, and increased vehicle wear and fuel use. These issues can be solved mainly by preventing vehicles from stopping at the intersections. This paper is focused on eliminating traffic stoppages at isolated intersections as an initial step in that direction. A method is proposed to eliminate stoppages by controlling the speed of vehicles approaching the isolated intersection. Vehicles can be either in a green interval or in a red interval. All vehicles in green interval are ordered to move on velocities above a predetermined minimum velocity $\left(V_{\min }\right)$ that allows vehicles to flow through the intersection safely. Other vehicles in red interval at the opposite direction are concurrently ordered to move at velocities under a predetermined maximum velocity $\left(V_{\max }\right)$ that forces vehicles to consume the typically red interval time before arriving at the intersection zone while they are in motion state. The state is then reversed where vehicles in red interval at first direction are ordered to move with $V_{\max }$ speed limit and vehicles in the green interval at the opposite direction are ordered to move on $V_{\min }$ and so forth. Fig. 1 depicts the operation of the proposed (Min-Max) traffic control system. To ensure feasibility, the proposed method is first proven with theoretical analysis and then verified using simulation modelling.

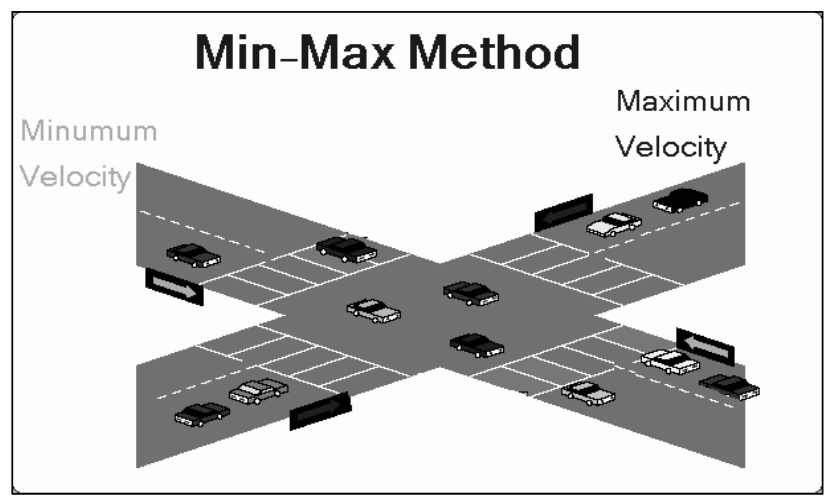

Figure 1: Min-Max method applied at an isolated intersection.

\subsection{Theoretical analysis of Min-Max method}

Fig. 2 shows the intersection dimensions used in the proposed Min-Max system.

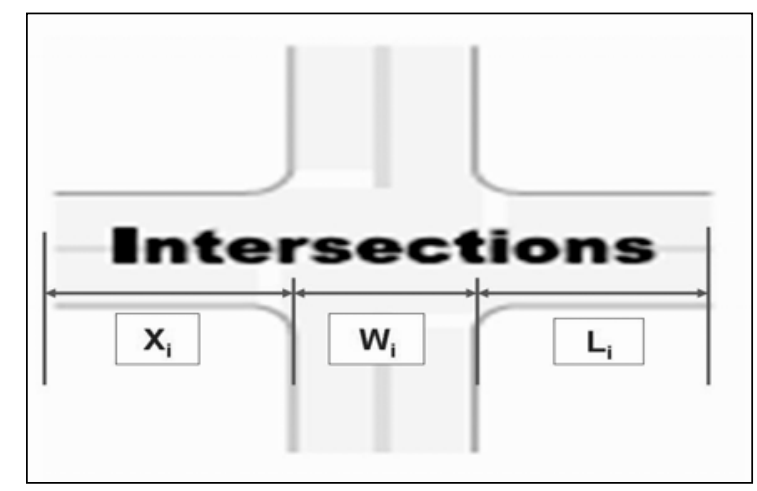

Figure 2: Intersection dimension parameters in the Min-Max method. 
By enhancing (3), another formula can be used for the yellow time in the conventional traffic signal system, based on Fig. 2:

$$
Y_{i}=\frac{\left(X_{i}+W_{i}+L_{i}\right)}{V_{0}}+t
$$

In (4), $X_{i}$ is determined from speed $v_{0}$ and time $t$. Fig. 3 shows the velocity relationship with the time in the conventional traffic system, where it can be noticed clearly that $v_{0}=0$ (indicating the occurrence of full stoppages).

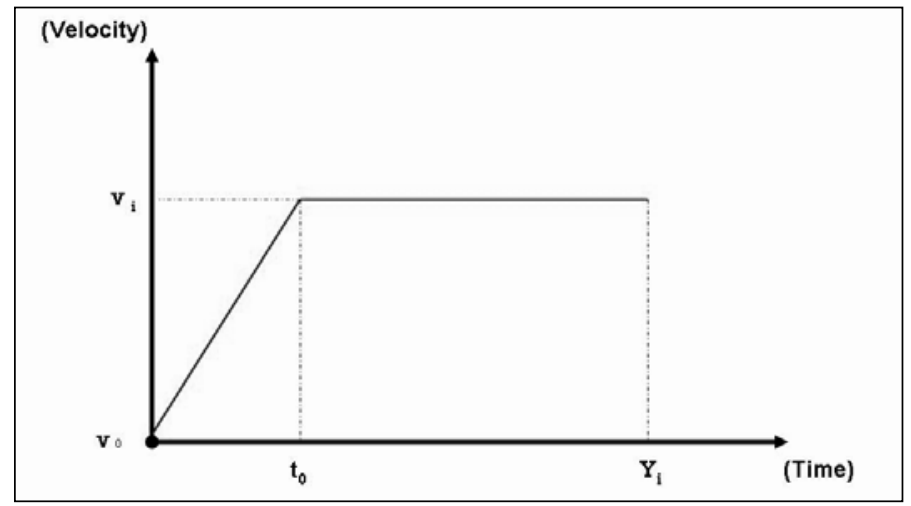

Figure 3: Velocity behavior in conventional traffic system.

For the proposed Min-Max method, and based on [19, 20], the yellow time is defined as follows to meet the requirement of no stoppages:

$$
Y_{i}=\frac{\left(X_{i}+W_{i}+L i\right)}{v_{f}}+\frac{\frac{1}{2}(v f-v 0)^{2}}{a(v f)}+t
$$

Fig. 4 shows the change of velocity with time in the proposed Min-Max method, where it can be noticed clearly that $v_{0} \neq 0$ (implying no stoppage of traffic at the intersection).

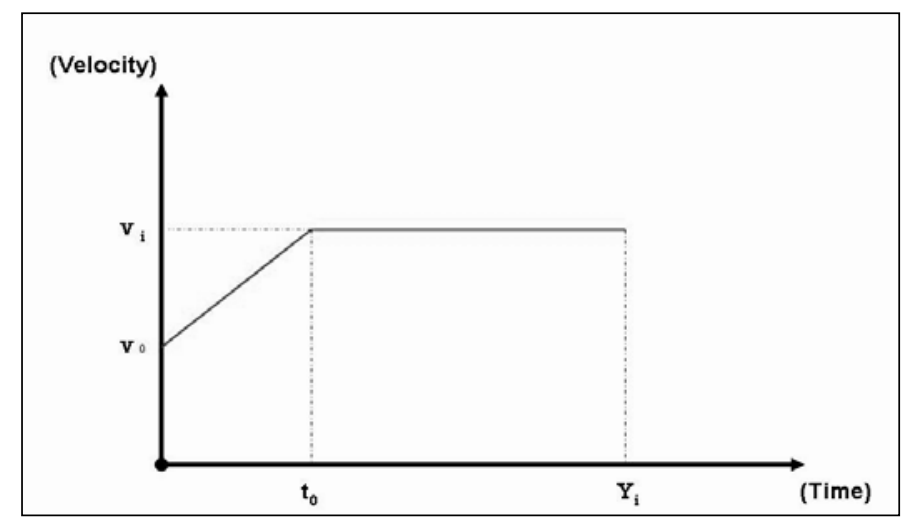

Figure 4: Velocity behavior the proposed Min-Max method.

\subsection{Alternating Min -Max speed limits}

Coordination of alternating Min-Max speed limits is essential to control the flow of vehicles and avoid collisions. As shown in Fig. 5(a), when switching from Min to Max velocity, the car in the front is ordered to move at Min velocity and the next car is ordered to move at Max 
velocity. Since $V_{\min }>V_{\max }$ in this case, the minimum difference between the two car's velocities is kept at $\left(V_{\min }-V_{\max }>0\right)$, which prevents collision.

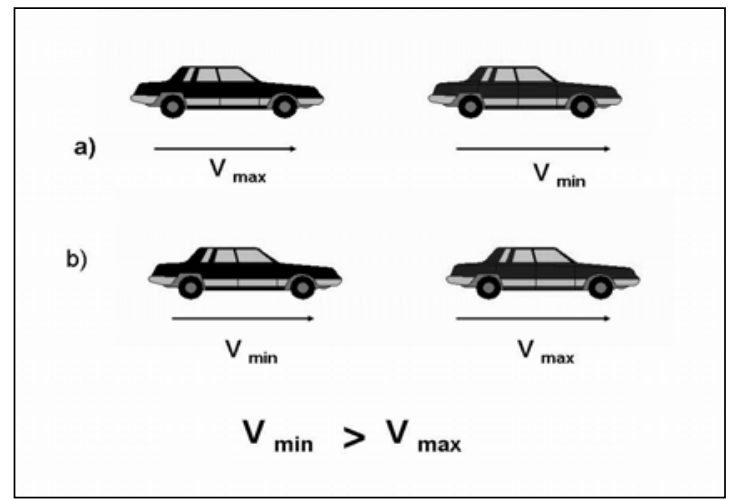

Figure 5: Alternating Min-Max speed limits.

Similarly, as shown in Figure 5(b), when switching from Max to Min velocity, the car in the front is ordered to move at Max velocity and the next car is ordered to move at Min velocity. However, since $V_{\min }>V_{\max }$, there will be a risk for collision to occur. Hence, a safety distance $\Delta X$ is maintained at each order of velocity change. After redefining the dimensions of the intersection as shown in Fig. $6, \Delta X$ is determined as follows:

$$
\Delta X=\left[\left[\frac{\left(V_{\min }-V_{\max }\right)}{V_{\max }}\right] X 2\right]+V_{\min }\left[\frac{\left(w_{i}+2 L\right)}{V_{\max }}+\frac{1}{2} \frac{\left(V_{\max }-v_{0}\right)^{2}}{a\left(V_{\max }\right)}\right]-\left[\left(w_{i}+L\right)+\frac{1}{2} \frac{\left(V_{\min }-v_{0}\right)^{2}}{a}\right]
$$

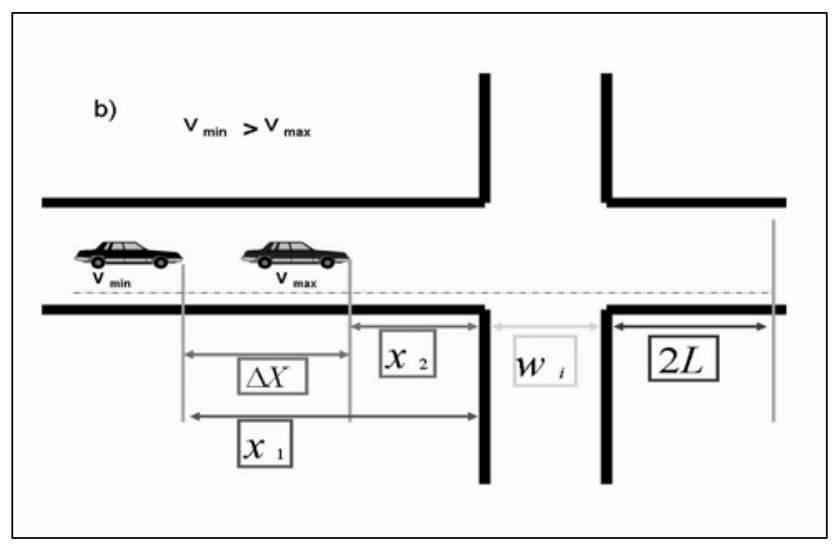

Figure 6: Redefined intersection dimensions to calculate $\Delta X$.

\subsection{Max and Min velocity formulas}

$V_{\max }$ and $V_{\min }$ represent the upper and lower limits forced on the vehicle approaching the intersection, respectively. These limits are assigned and alternated between the two sides of the roads. To assign values for $V_{\min }$ and $V_{\max }$, we first define the green (G) and red (R) interval times for the two speeds. Based on [3], G and R are defined as follows:

$$
\begin{array}{ll}
\frac{G_{1}}{G_{2}}=\frac{N_{1}\left(S_{1}\right)}{N_{2}\left(S_{2}\right)} & G_{1}=\frac{C-\left(Y_{1}+Y_{2}\right)}{\left(1+\frac{N_{2}}{N_{1}}\right)} \quad G_{2}=C-\left(Y_{1}+Y_{2}\right)-G_{1} \\
R_{1}=G_{2}+Y_{2} & R_{2}=G_{1}+Y_{1}
\end{array}
$$


Using 7(a) and 7(b), $V_{\min }$ and $V_{\max }$ are derived for the two sides of the road using the dimensions shown in Fig. 7 as follows:

$$
\begin{array}{ll}
V_{1(\text { max })}=\frac{X_{1}}{R_{2}} & V_{2(\max )}=\frac{X_{2}}{R_{1}} \\
V_{2(\min )} \geq \frac{X_{2}+W_{2}+L_{2}+\Delta X_{2}}{G_{2}} & V_{1(\min )} \geq \frac{X_{1}+W_{1}+L_{1}+\Delta X_{1}}{G_{1}}
\end{array}
$$

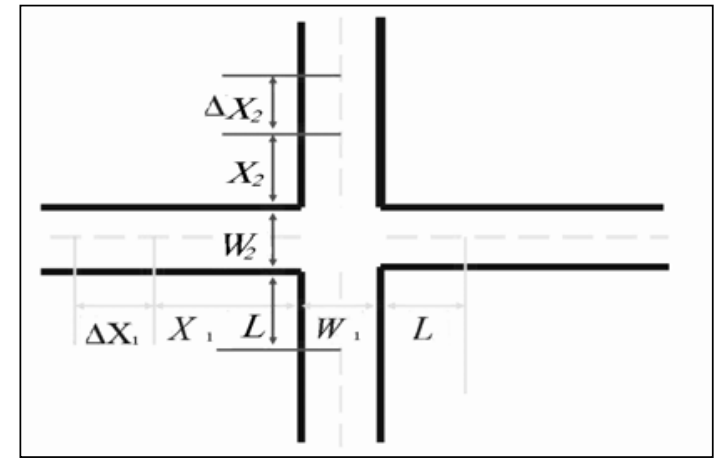

Figure 7: Road dimensions for setting $V_{\min }$ and $V_{\max }$.

A problem arises for the flow of in-between vehicles when alternating $V_{\max }$ and $V_{\min }$. As shown in Fig. 8, some in-between vehicles may be ordered to switch speed twice from $V_{\min }$ to $V_{\max }$ and then from $V_{\max }$ to $V_{\min }$ and some may miss the two speed-change signals.

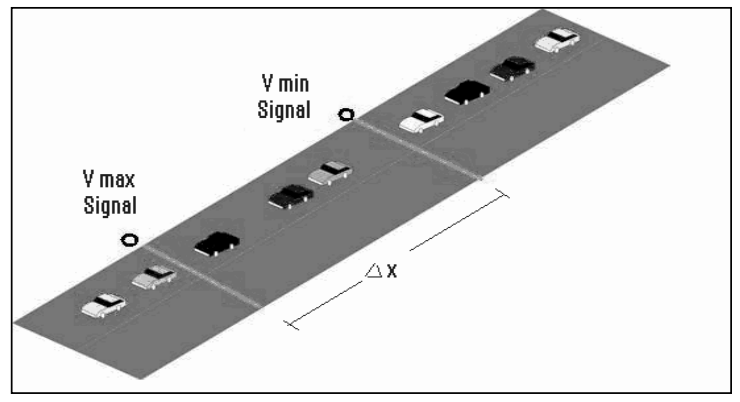

Figure 8: Elapsed time between $\mathrm{V}_{\max }$ and $\mathrm{V}_{\text {min. }}$.

It is therefore needed to prevent the simultaneous speed switches by creating an elapsed time (offset) so that each vehicle receives one and only one order to switch speed before arriving at the intersection. To this end, the operation of $V_{\max }$ sign is delayed when switching speed from $V_{\min }$ to $V_{\max }$ by a predetermined period of time $(T)$ as follows:

$$
T_{\text {between }}=\frac{\Delta X}{v_{0}}
$$

Where $\Delta X$ is the distance between two speed signs and $v_{0}$ is the velocity of vehicle before reaching the first speed sign. Similarly, when switching speed from $V_{\max }$ to $V_{\min }$, we delay the operation of $V_{\min }$ sign by a predetermined period of time. This prevents in-between vehicles from flowing without receiving an order of speed change. The elapsed time in this situation is defined as follows:

$$
\text { Tbetween }=\frac{\Delta X}{v_{0}}+\frac{\frac{1}{2}\left(V \min -v_{0}\right)^{2}}{a(V \min )}
$$

Where $a$ is the deceleration/acceleration rate of clearing vehicle. 


\section{TRAFFIC SIMULATION MODELS}

DES modelling is utilized to animate the operation of the proposed Min-Max method, verify its theoretical analysis, and compare its performance to the conventional traffic lights system. Since conventional traffic lights are of proven functionality in the real-world, the simulationbased comparison is a valid approach under the same traffic conditions and parameters described in [3]. The model is also used as a platform to test the possibilities and determine the limitations of controlling the real traffic flow for such conditions. To this end, two simulation models were developed using AutoMod 3D simulation package; one for conventional traffic light system and another for the proposed Min-Max traffic control system. AutoMod is simulation software that is primarily used in industrial applications such as material handling systems, assembly lines, and production controls. In this paper, AutoMod is mainly used due to its relative flexibility in developing and debugging the traffic control logic of Min-Max method and due to its 3D animation capability in displaying and validating traffic flow. Details of AutoMod software can be found in [21].

Steps in a traffic simulation study with AutoMod are similar to typical simulation studies. As discussed in [12], the conceptual model is first developed using the structure and theoretical basis of the of the traffic control system. The model is then constructed using the software modules. Simultaneously, model data and assumptions are set based on [3] including pattern of vehicles flow, road and intersection dimensions, travel speed, and signalling intervals. The conveyor module in AutoMod is particularly utilized in this study to model traffic flow (i.e., conveyer sections as roads, loads as vehicles, and stations as control points) along with AutoMod's flexible editing and programming platform. The movement of loads is identical to two-phase system rules. The roads are controlled using conveyer attributes of length, width, velocity, acceleration, direction, and other parameters. To create traffic signals that allow vehicles to move during green interval and to stop during red interval, a dummy AutoMod resource is developed with zero processing time, constant mean time between failure (MTBF) during green interval, and constant mean time to repair (MTTR) during red interval. The logic for speed setting and switching is programmed and a set of measures of performance (MOP) is defined using model counters and variables. The built DES model is then validated and verified as discussed in [22]. After setting model run controls (warm-up period, run length, and number of replications), the model is run and simulation results, in terms of MOP values, are collected and analyzed. Based on analysis, simulation results are then documented and reported.

\subsection{Simulation results for conventional traffic signals}

The first AutoMod model is developed to mimic the operation of conventional traffic signals at an isolated intersection. The procedure discussed earlier is followed in developing, verifying, and validating the traffic simulation model. Several input parameters are predetermined and set with initial values in the AutoMod model of the conventional traffic signals. These model inputs are shown in Table I. At the end of simulation runs, AutoMod displays summary reports and business graphs for each model-defined process, queue, resource, section, station, and load. The model is also set to produce specific statistics to summarize the performance of traffic flow at the isolated intersection. Model outputs and collected statistics from simulation results of conventional system are shown in Table II. These statistics are used in the model to dynamically report a set of five MOPs. These measures will be used to compare the performance of the proposed system compared to the conventional system under the same model parameters and running conditions. These MOPs include average travel time, average delay time, average travel speed, average number of stops made, and the average number of vehicles at the isolated intersection. 
Table I: Simulation inputs for the conventional system.

\begin{tabular}{|l|l|}
\hline \multicolumn{1}{|c|}{ Model Input } & \multicolumn{1}{c|}{ Value } \\
\hline Road travel speed & $16.67 \mathrm{~m} / \mathrm{sec}$ \\
\hline Green time interval (MTBF) & $30 \mathrm{sec}$ \\
\hline Red time interval (MTTR) & $35 \mathrm{sec}$ \\
\hline Cycle time of traffic signal & $70 \mathrm{sec}$ \\
\hline Lane width & $3.66 \mathrm{~m}$ \\
\hline Travel distance to pass the intersection & $1829 \mathrm{~m}$ \\
\hline Vehicles arrival distribution & Exponential \\
\hline Mean time between arrivals (1/ $\lambda$ ) & $4 \mathrm{sec}$ \\
\hline Simulation run time & $12 \mathrm{hrs}$ \\
\hline Number of simulation replicates & 5 \\
\hline
\end{tabular}

Table II: Simulation results for conventional system.

\begin{tabular}{|l|l|}
\hline \multicolumn{1}{|c|}{ Simulation Output (Statistic) } & Value \\
\hline Total number of simulated vehicles at the intersection & 10934 Vehicles \\
\hline Average number of vehicles at the intersection & 30.11 Vehicles \\
\hline Maximum number of vehicles at the intersection & 54 Vehicles \\
\hline Minimum number of vehicles at the intersection & 12 Vehicles \\
\hline Average vehicle travel time when passing intersection & $118.98 \mathrm{sec}$ \\
\hline Average delay time per vehicle compared to free flow & $9.49 \mathrm{sec}$ \\
\hline Total number of vehicles stopped on red signal & 5873 Vehicles \\
\hline Average number of vehicles waiting on red signal & 2.39 Vehicles \\
\hline Maximum number of vehicle waiting on red signal & 18 Vehicles \\
\hline Minimum number of vehicle waiting on red signal & 0 Vehicles \\
\hline Average vehicle waiting time on red signal & $17.62 \mathrm{sec}$ \\
\hline Percentage of vehicles stopped on red signal & $53.87 \%$ \\
\hline
\end{tabular}

\subsection{Simulation results for the proposed system}

Model inputs for the proposed Min-Max system simulation are shown in Table III. Simulation results of the proposed system are shown in Table IV.

Table III: Simulation inputs for the proposed Min-Max system.

\begin{tabular}{|l|l|}
\hline Model Input & Value \\
\hline Road original travel speed & $16.67 \mathrm{~m} / \mathrm{sec}$ \\
\hline Road sign-set Max speed $\left(V_{\max }\right)$ & $9.15 \mathrm{~m} / \mathrm{sec}$ \\
\hline Road sign-set Min speed $\left(V_{\min }\right)$ & $24.4 \mathrm{~m} / \mathrm{sec}$ \\
\hline Travel distance at $V_{\max }$ & $445.0 \mathrm{~m}$ \\
\hline Travel distance at $V_{\min }$ & $853.5 \mathrm{~m}$ \\
\hline Vehicle Acceleration/Deceleration & $4.572 \mathrm{~m} / \mathrm{sec}^{2}$ \\
\hline Cycle time (switching speeds) & $76 \mathrm{sec}$ \\
\hline Lane width & $3.66 \mathrm{~m}$ \\
\hline Travel distance to pass the intersection & $1829 \mathrm{~m}$ \\
\hline Vehicles arrival distribution & Exponential \\
\hline Mean time between arrivals $(1 / \lambda)$ & $4 \mathrm{sec}$ \\
\hline Simulation run time & $12 \mathrm{hrs}$ \\
\hline Number of simulation replications & 5 \\
\hline
\end{tabular}


Table IV: Simulation results for the proposed system.

\begin{tabular}{|l|l|}
\hline \multicolumn{1}{|c|}{ Model Output (Statistic) } & \multicolumn{1}{c|}{ Value } \\
\hline Total number of simulated vehicles at the intersection & 10656 Vehicles \\
\hline Average number of vehicles at the intersection & 27.67 Vehicles \\
\hline Maximum number of vehicles at the intersection & 52 Vehicles \\
\hline Minimum number of vehicles at the intersection & 11 Vehicles \\
\hline Average vehicle travel time when passing intersection & 112.17 sec \\
\hline Average delay time per vehicle compared to free flow & 3.08 sec \\
\hline Total number of vehicles stopped at intersection & 0 Vehicles \\
\hline
\end{tabular}

\section{DISCUSSION OF RESULTS}

As shown in Table V, a comparison is held between the simulation results of the conventional and the proposed systems based on five MOPs. The selected MOPs highlight the benefits of the proposed system at isolated intersections in terms of the overall traffic flow. Using a theoretical travel speed of $16.67 \mathrm{~m} / \mathrm{sec}$, the conventional system resulted in an average vehicle speed of $15.37 \mathrm{~m} / \mathrm{sec}$. Under the same conditions, the average vehicle speed in the proposed system is higher $16.30 \mathrm{~m} / \mathrm{sec}$. Consequently, the average travel time per vehicle in conventional system is $118.98 \mathrm{sec}$ compared to $112.17 \mathrm{sec}$ at the same travel distance (1829 $\mathrm{m})$ in the proposed system. The two measures indicate a better flow rate in the proposed system with a 5.72 reduction in average vehicle travel time.

Table V: MOP-based comparison of conventional and proposed systems.

\begin{tabular}{|c|l|l|l|c|}
\hline No. & $\begin{array}{c}\text { MOP } \\
\text { (Average per Vehicle) }\end{array}$ & $\begin{array}{c}\text { Conventional } \\
\text { System }\end{array}$ & $\begin{array}{c}\text { Proposed } \\
\text { System }\end{array}$ & $\begin{array}{c}\text { \% } \\
\text { Improvement }\end{array}$ \\
\hline 1 & Travel time & $118.98 \mathrm{sec}$ & $112.18 \mathrm{sec}$ & $5.72 \%$ \\
\hline 2 & Vehicle delay time & $9.49 \mathrm{sec}$ & $3.08 \mathrm{sec}$ & $67.55 \%$ \\
\hline 3 & Travel speed & $15.37 \mathrm{~m} / \mathrm{sec}$ & $16.30 \mathrm{~m} / \mathrm{sec}$ & $5.72 \%$ \\
\hline 4 & Number of stops & $0.54 \mathrm{stop}$ & 0 stop & --- \\
\hline 5 & Vehicles at intersection & $30.11 \mathrm{Veh}$. & $27.67 \mathrm{Veh}$. & $8.12 \%$ \\
\hline
\end{tabular}

The average vehicle waiting (delay) time depicts the time difference between the actual average travel time and the free moving time (i.e., vehicles are delayed if they cannot travel at the free flow speed). In the conventional system, vehicles are delayed when forced to stop at the red traffic signal. In the proposed system, vehicles are delayed when forced to reduce speed below or equal $V_{\max }$. The average vehicle delay in conventional system is $9.49 \mathrm{sec}$ compared to $3.08 \mathrm{sec}$ at the same travel distance in the proposed system. This $67.55 \%$ reduction in average vehicles delay time results in better flow and less congestion.

In terms of average number of stops per vehicle, the conventional system reports 0.537 stops per vehicle with an average waiting time of $17.62 \mathrm{sec}$. This is compared to 0 stops per vehicle in the proposed system out of over the 10656 vehicles passed during the 12-hour simulation period. This validates the results of the theoretical Min-Max model in eliminating vehicle stoppages ( $0 \%$ stopped) at isolated intersections.

Finally, in terms of traffic density, the average number of vehicles in the conventional system (within the $1829 \mathrm{~m}$ ) is 30.11 vehicles compared to 27.67 vehicles in the proposed system (i.e., $8.12 \%$ reduction). This indicates better capacity and less congestion and traffic density in the proposed system. The maximum number of vehicles in the conventional system has reached 54 compared to 52 in the proposed system. 


\subsection{Real-world traffic limitations}

Adopting the proposed Min-Max method to control the real-world traffic has high potential in improving the overall traffic flow, as seen from the results of simulation. However, since no data is available from a real-world experiment, it is imperative to address possibilities and limitations. Adopting the approach to replace conventional traffic signals mainly requires two electronic velocity signals separated by $\Delta X$ distance at each direction of the intersection; one for $V_{\max }$ and another for $V_{\min }$. Conditions for practical use mainly require alarming vehicles approaching the intersection, driver's compliance with the posted speeds at the signs, and clear safety warnings for pedestrians of no stoppages at the intersection. The flexibility of the proposed Min-Max method makes it adaptable and consequently functional at different speeds and road conditions (e.g., slow traffic due to construction or weather conditions). The proposed system allows for setting the values of $V_{\max }$ and $V_{\min }$, timing switching signals, changing the locations of velocity signals, and adapting system to various speed limits. Simulation experiments showed that the functionality is maintained when changing simulation inputs and road conditions. Safety allowance should be also applied for the speed limits and travel distances based on prediction of driver's non-compliance behaviour. It is highly recommended, however, to test the system first at an isolated intersection with trained drivers before adopting.

One of the major problems in traffic engineering comes from the wide range of variability in driver response to the traffic environment and high percentage of speed non-compliance. Forcing drivers to comply with the Min-Max speed limits is very much similar to forcing drivers to stop at red lights and to drive at posted speeds on the highway. One recommended technique is "learning by habit" where drivers are allowed to try the new system at selected intersections and compare the free flow at $V_{\max }$ or $V_{\min }$ to stop-and-go traffic pattern. As drivers feel the difference, their compliance improves. Another technique is Intelligent Vehicle Highway System (IVHS) in which vehicles communicate with the road and driving is automated using equipment in the vehicle and sensors on the road and through GPS (Global Positioning System).

\section{CONCLUSION}

This paper proposed a traffic control system to eliminate vehicle stoppages at isolated signalised intersections. The paper has presented the theoretical analysis of the proposed MinMax method, derived values for $V_{\max }$ to $V_{\min }$, and set controls for alternating vehicle speeds at the two sides of the intersection. The results of the theoretical analysis were verified using Discrete Event Simulation (DES) and compared to the conventional traffic light signals. Traffic modelling with DES allowed for deploying flow logic, animating traffic flow, verifying the non-stop functionality at the intersection, and estimating values for key Measures of Performance (MOP). Simulation results demonstrated the advantages of the proposed Min-Max method in eliminating stoppages at the isolated intersection, reducing traffic congestion, reducing vehicles delay, and improving vehicles flow. Other benefits from eliminating vehicles stoppages include a reduction in fuel consumption, air pollution, braking, and crash. The paper also addressed the possibilities and limitations of adopting the proposed system in controlling real-world traffic. The flexibility of the proposed system and the relatively low cost of needed control equipment encourage the applicability of the Min-Max system to replace traffic lights at isolated intersections. System deployment should be tested for real traffic and combined with proper safety measures. Further research can be directed to utilizing DES in incorporating measures for protecting pedestrians, handling arterial roads, coordinating adjacent intersections, testing the three-phase and four-phase signalling system, and assuring drivers compliance. 


\section{REFERENCES}

[1] Gartner, N. H.; Messer, C. J.; Rathi, A. K. (Eds.) (1999). Traffic Flow Theory: A State-of-the-Art Report, Transportation Research Board, Washington, DC

[2] Garber, N.; Hoel, L. (2002). Traffic and Highway Engineering, $3^{\text {rd }}$ Edition, The Wadsworth Group

[3] Yu, J. (1982). Transportation Engineering: Introduction to planning, design, and operation, Elsevier North Holland, The Netherlands

[4] Bruce, M. G.; Gruner, P. W. (2006).Continuous Flow Intersections, CE News, Vol. 17, No. 12, Zweig White Information Services

[5] Pitaksringkarn, J. P. (2005). Measures of Effectiveness for Continuous Flow Intersection: A Maryland Intersection Case Study, ITE 2005 Annual Meeting and Exhibit Compendium of Technical Papers, Institute of Transportation Engineers, Australia Road Research Board

[6] Berkowitz, C.; Mier, F.; Walter, C. E.; Bragdon, C. (1997). Continuous flow intersection: An intelligent transportation solution, $67^{\text {th }}$ annual meeting, Institute of Transportation Engineers

[7] Jiang, Y.; Li, S.; Shamo, D. E. (2006), A platoon-based traffic signal timing algorithm for majorminor intersection types, Transportation Research, Part B 40, 543-562

[8] FHWA (1998). Traffic Software Integrated System: Version 4.2 User's Guide, US Department of Transportation, Federal Highway Administration. Washington, DC

[9] Ishaque, M. M.; Noland, R. B. (2007). Trade-offs between vehicular and pedestrian traffic using micro-simulation methods, Transport Policy, Vol. 14, 124-138

[10] Jones Jr., S. J.; Sullivan, A. J.; Cheekoti, N.; Anderson, M. D. (2004). Traffic simulation software comparison study, UTCA Report 02217, University Transportation Centre for Alabama, The University of Alabama in Huntsville

[11] Kaseko, M. S. (2002). Comparative evaluation of simulation software for traffic operations, University of Nevada, Las Vegas, Nevada Department of Transportation

[12] Law, M.; Kelton, D. (2000). Simulation Modeling and Analysis, $3^{\text {rd }}$ ed., McGraw-Hill, New York

[13] Banks, J. (ed.) (1998). Handbook of Simulation: Principles, Methodology, Advances, Applications, and Practice, John Wiley

[14] Banks, J.; Carson II, J.; Nelson, B.; Nicol, D. (2001). Discrete-Event System Simulation, $3^{\text {rd }}$ ed., Prentice Hall, Upper Saddle River, New Jersey

[15] Chao-Yu, C.; Chung-Ho, C.; Ming-Hsien, C. L. (2001). Application of computer simulation to the design of a traffic signal timer, Computers \& Industrial Engineering, Vol. 39, Issue 1-2, 8194

[16] Dotoli, M.; Fanti, M. P. (2006). An urban traffic network model via coloured timed Petri nets, Control Engineering Practice, Vol. 14, 1213-1229

[17] Mohy El-Din, M.; El-Araby, K. (1999). A robust dynamic highway traffic simulation model, Computers \& Industrial Engineering, Vol. 37, Issue 1-2, 189-193

[18] Institute of Transportation Engineers (2004). Annual Meeting and Exhibit. Briefing Paper on Intersection Traffic Control, Way finding Cues at Intersections Workshop

[19] Serway, R. (1990). Physics for Scientists and Engineers with Modern Physics, $3^{\text {rd }}$ ed., Saunders Golden Sunburst, Florida

[20] Hibbeler, R. (2001). Engineering Mechanics Dynamics, $9^{\text {th }}$ ed., Prentice Hall, Upper Saddle River, New Jersey

[21] Banks, J. (2004). Getting Started with AutoMod, $2^{\text {nd }}$ ed., John Wiley \& Sons, Inc, New York

[22] Law, A.; McComas, M. G. (2001). How to build valid and credible simulation models, Proceedings of the 2001 Winter Simulation Conference, 22-28 